\title{
A Comparison between Modified Sumudu Decomposition Method and Homotopy Perturbation Method
}

\author{
Shams A. Ahmed ${ }^{1,2}$ \\ ${ }^{1}$ Department of Mathematic, Faculty of Sciences and Arts-Tubarjal, Aljouf University, Al Jawf, KSA \\ ${ }^{2}$ Department of Mathematics, Faculty of Mathematics and Computer Sciences, University of Gezira, Wad Madani, Sudan \\ Email:Shamsalden20@hotmail.com
}

How to cite this paper: Ahmed, S.A. (2018) A Comparison between Modified Sumudu Decomposition Method and Homotopy Perturbation Method. Applied Mathematics, 9, 199-206.

https://doi.org/10.4236/am.2018.93014

Received: January 27, 2018

Accepted: March 16, 2018

Published: March 19, 2018

Copyright $\odot 2018$ by author and Scientific Research Publishing Inc. This work is licensed under the Creative Commons Attribution International License (CC BY 4.0).

http://creativecommons.org/licenses/by/4.0/

\begin{abstract}
In this paper, we present a comparative study between the modified Sumudu decomposition method (MSDM) and homotopy perturbation method (HPM). The study outlines the important features of the two methods. The analysis will be explained by discussing the nonhomogeneous Kortewege-de Vries $(\mathrm{KdV})$ problems.
\end{abstract}

\section{Keywords}

The Sumudu Decomposition Method, The Homotopy Perturbation Method, Kortewege-de Vries (KdV) Problems

\section{Introduction}

This paper outlines effective comparison between two powerful methods that were newly advanced. The first method is modified form of the Sumudu decomposition method (MSDM) developed by Devedra Kumar [1] and used in [2] [3] [4] [5] [6] among many others. The second method is homotopy perturbation method (HPM) developed by He [7] and used from many researchers [8] [9] [10]. The two methods give quickly convergent series and are successfully used to solve a large number of linear and nonlinear problems.

In the present study, we consider the nonhomogeneous $\mathrm{KdV}$ equation of the following type:

$$
U_{t}+a U U_{x}+b U_{x x x}=f(x, t),
$$

with the initial condition;

$$
U(x, 0)=g(x) .
$$


where $a$ and $b$ are constants.

The nonlinear KdV Equation (1) is an important mathematical model with wide applications in quantum mechanics and nonlinear optics. The KdV equation has several applications to physical problems. It approximately describes the evolution of long water waves. In addition, it is used in various fields such as, shallow water waves, acoustic waves in plasma, and long internal waves in a density.

The main objective of this contribution is to introduce a comparative study to solve KdV equation by using two of the most recently developed methods, the (MSDM), and the (HPM). The two methods accurately compute the solution in a series form or in an exact form.

\section{Analysis of Numerical Methods}

\subsection{Basic Idea of the MSDM}

To illustrate the basic idea of this method, we consider a general non-homogeneous partial differential equation with the initial conditions of the form:

$$
\begin{aligned}
& D U(x, t)+R U(x, t)+N U(x, t)=g(x, t) \\
& U(x, 0)=h(x), U_{t}(x, 0)=f(x) .
\end{aligned}
$$

where $D$ is the second order linear differential operator $D=\frac{\partial^{2}}{\partial t^{2}}, R$ is other linear differential operator of less order than $D, N$ represent the general nonlinear operator and $g(x, t)$ is the source term.

Taking the Sumudu transform of both sides of Equation (3), we get:

$$
S[L U(x, t)]+S[R U(x, t)]=S[g(x, t)] ;
$$

Using the differentiation property of the Sumudu transform and given initial conditions, we have:

$$
S[U(x, t)]=u^{2} S[g(x, t)]+h(x)+u f(x)-u^{2} S[R U(x, t)+N U(x, t)] .
$$

If we apply the inverse operator $S^{-1}$ to both sides of the Equation (5), we obtain:

$$
U(x, t)=G(x, t)-S^{-1}\left[u^{2} S[R U(x, t)+N U(x, t)]\right] .
$$

where $G(x, t)$ represents the term arising from the source term and the prescribed initial conditions. Now, apply the Adomain decomposition method [11] [12] [13] [14]:

$$
U(x, t)=\sum_{n=0}^{\infty} U_{n}(x, t),
$$

The nonlinear term can be decomposed as:

$$
N U(x, t)=\sum_{n=0}^{\infty} A_{n}(U),
$$

For some Adomian polynomials $A_{n}(U)$ that are given by: 


$$
A_{n}\left(U_{0}, U_{1}, U_{2}, \cdots, U_{n}\right)=\frac{1}{n !} \frac{\mathrm{d}^{n}}{\mathrm{~d} \lambda^{n}}\left[N\left(\sum_{n=0}^{\infty} \lambda^{n} U_{n}\right)\right]_{\lambda=0}, n=0,1,2, \cdots
$$

Substituting Equation (7) and Equation (8) in Equation (6), we get:

$$
\sum_{n=0}^{\infty} U_{n}(x, t)=G(x, t)-S^{-1}\left[u^{2} S\left[R \sum_{n=0}^{\infty} U_{n}(x, t)+\sum_{n=0}^{\infty} A_{n}(U)\right]\right] .
$$

Accordingly, the formal recursive relation is defined by:

$$
\begin{aligned}
& U_{0}(x, t)=G(x, t), \\
& U_{k+1}(x, t)=-S^{-1}\left[u^{2} S\left[R U_{k}+A_{k}\right]\right], k \geq 0 .
\end{aligned}
$$

The modified Sumudu decomposition method introduces a slight variation to the recursive relation (10) that will lead to the determination of the components of $U$ in a faster and easier way [15] [16]. For specific cases, the function $G(x, t)$ can be set as the sum of two partial functions, namely $f_{1}(x, t)$ and $f_{2}(x, t)$. In other words, we can set:

$$
G(x, t)=f_{1}+f_{2}
$$

Using (11), we introduce a qualitative change in the formation of recursive relation (10). The modified recursive relation can be identified by:

$$
\begin{gathered}
U_{0}=f_{1}, \\
U_{1}=f_{2}-S^{-1}\left[u^{2} S\left[R U_{0}+A_{0}\right]\right], \\
U_{k+1}=-S^{-1}\left[u^{2} S\left[R U_{k}+A_{k}\right]\right] ; k \geq 1
\end{gathered}
$$

The solution through the modified Sumudu decomposition method is highly depend upon the choice of $f_{1}(x, t)$ and $f_{2}(x, t)$.

\subsection{Basic Idea of HPM}

Consider the following general nonlinear differential equation,

$$
u-N(u)=f \text {, }
$$

where $N$ is a nonlinear operator from Hilbert space $H$ to $H, u$ is an unknown function, and $f$ is a known function in $H$.

The homotopy perturbation method $u$ as a series with components $u_{n}$, and $N(u)$ as a series with components $H_{n}$, homotopy polynomials, which can be calculated using the formula:

$$
H_{n}=\left.\frac{1}{n !} \frac{\mathrm{d}^{n}}{\mathrm{~d} \lambda^{n}} N\left(\sum_{i=0}^{\infty} u_{i} \lambda^{i}\right)\right|_{\lambda=0}
$$

To illustrate the homotopy perturbation method (HPM), we consider (15) as;

$$
L(v)=v(x)-f(x)-N(v)=0
$$

with solution $u(x)$. As a possible remedy, we can define homotopy $H(v, p)$ as follows:

$$
H(v, 0)=F(v), H(v, 1)=L(v)
$$

where $F(v)$ is an integral operator with known solution $v_{0}$, which can be ob- 
tained easily. Typically, we may choose a convex homotopy in the form;

$$
H(v, p)=(1-p) F(v)+p L(v)=0
$$

and continuously trace an implicitly defined curve from a starting point $H\left(v_{0}, 0\right)$ to a solution function $H(u, 0)$. The embedding parameter $p$ monotonically increase from zero to unit as the trivial problem $F(v)=0$ is continuously deformed to original problem $L(v)=0$.

$$
v=v_{0}+p v_{1}+p^{2} v_{2}+p^{3} v_{3}+\cdots
$$

when $p \rightarrow 1$, Equation (18) corresponds to Equation (17) and Equation (19) becomes the approximate solution of Equation (17), i.e.

$$
v=\lim _{p \rightarrow 1} v_{0}+v_{1}+v_{2}+v_{3}+\cdots
$$

\section{Application}

In this section, we demonstrate the analysis of two methods by applying two methods to the following two Kortewege-de Vries (KdV) partial differential equations.

Example 1: Consider the following inhomogeneous nonlinear $\mathrm{KdV}$ equation [17]:

$$
u_{t}+u u_{x}+u_{x x x}=\sin x+t \cos x(t \sin x-1)
$$

With the initial condition:

$$
u(x, 0)=0
$$

\section{1) Using HPM}

To solve Equations (21)-(22) by homotopy perturbation method, we construct the following homotopy:

$$
\frac{\partial v}{\partial t}-\frac{\partial u_{0}}{\partial t}=p\left(-u \frac{\partial u}{\partial x}-\frac{\partial^{3} u}{\partial x^{3}}+\sin x+t \cos x(t \sin x-1)-\frac{\partial u_{0}}{\partial t}\right)
$$

Assume the solution of Equation (23) to be in the form:

$$
v=v_{0}+p v_{1}+p^{2} v_{2}+p^{3} v_{3}+\cdots
$$

Substituting (24) into (23) and comparing coefficients of terms with identical powers of $p$, leads to:

$$
\begin{gathered}
p^{0}: \frac{\partial v_{0}}{\partial y}-\frac{\partial u_{0}}{\partial y}=0 \\
p^{1}: \frac{\partial v_{1}}{\partial t}=-u_{0} \frac{\partial u_{0}}{\partial x}-\frac{\partial^{3} u_{0}}{\partial x^{3}}+\sin x+t^{2} \cos x \sin x-t \cos x-\frac{\partial u_{0}}{\partial t}
\end{gathered}
$$

The given initial value admits the use of:

$$
u_{0}(x, 0)=0
$$

The solution reads:

$$
u_{0}(x, t)=0
$$




$$
\begin{gathered}
u_{1}(x, t)=t \sin x+\frac{t^{3}}{3} \sin x \cos x-\frac{t^{2}}{2} \cos x \\
u_{2}(x, y)=-\frac{t^{3}}{3} \sin x \cos x+\frac{t^{2}}{2} \cos x-\frac{t^{4}}{3} \sin ^{2} x-\frac{t^{4}}{3} \sin ^{3} x+\cdots
\end{gathered}
$$

Examining the components $u_{1}$ and $u_{2}$ in Equation (29) and Equation (30), we can easily observe that the last two terms in $u_{1}$ and the first two terms in $u_{2}$ are the self-canceling (noise terms) [18]. Hence, the non-noise terms in $u_{1}$ yields the exact solution of Equations (21)-(22), given by:

$$
u(x, t)=t \sin x \text {. }
$$

\section{Notes on HPM:}

From the previous analysis, we can observe that:

- HPM can be applied it to various nonlinear problems. The main disadvantage is that we should suitably choose an initial guess.

- HPM needs some modification to the rapid convergence of the series solution.

To overcome these disadvantages of HPM, the following ADSTM method is suggested.

2) Using MSDM

By taking Sumudu transform for (21) and using (22) we obtain:

$$
S[U(x, t)]=u \sin x+2 u^{3} \cos x \sin x-u^{2} \cos x-u S\left[U U_{x}+U_{x x x}\right] .
$$

Applying $S^{-1}$ to both sides of (32) we obtain;

$$
U(x, t)=t \sin x+\frac{t^{3}}{3} \cos x \sin x-\frac{t^{2}}{2} \cos x-S^{-1}\left[u S\left[U U_{x}+U_{x x x}\right]\right] .
$$

Substituting;

$$
U(x, t)=\sum_{n=0}^{\infty} U_{n}(x, t) ;
$$

And the nonlinear terms of;

$$
U U_{x}=\sum_{n=0}^{\infty} A_{n} .
$$

Into (33) gives;

$$
\begin{aligned}
\sum_{n=0}^{\infty} U_{n}(x, t)= & t \sin x+\frac{t^{3}}{3} \cos x \sin x-\frac{t^{2}}{2} \cos x \\
& -S^{-1}\left[u S\left[\sum_{n=0}^{\infty} A_{n}+\left(\sum_{n=0}^{\infty} U_{n}(x, t)\right)_{x x x}\right]\right]
\end{aligned}
$$

This gives the modified recursive relation;

$$
\begin{gathered}
U_{0}(x, t)=t \sin x \\
U_{1}(x, t)=\frac{t^{3}}{3} \cos x \sin x-\frac{t^{2}}{2} \cos x-S^{-1}\left[u S\left[A_{0}+\left(U_{0}\right)_{x x x}\right]\right], \\
U_{k+1}(x, t)=-S^{-1}\left(A_{k}+U_{k}\right), k \geq 1 .
\end{gathered}
$$

The first few of the components are given by; 


$$
\begin{gathered}
U_{0}(x, t)=t \sin x, \\
U_{1}(x, t)=\frac{t^{3}}{3} \cos x \sin x-\frac{t^{2}}{2} \cos x-S^{-1}\left[u S\left[A_{0}+\left(U_{0}\right)_{x x x}\right]\right]=0, \\
U_{k+1}(x, t)=0, \quad k \geq 1 .
\end{gathered}
$$

The solution in a closed form is given by;

$$
U(x, t)=t \sin x .
$$

Example 2: Consider the following inhomogeneous nonlinear $\mathrm{KdV}$ equation [17]:

$$
u_{t}+u u_{x}+u_{x x x}=(x t+\sin x)(t+\cos x)+x+\cos x ;
$$

With the initial condition:

$$
u(x, 0)=\sin x
$$

\section{1) Using HPM}

Using homotopy perturbation method like in Example 1, we obtain the following components:

$$
\begin{gathered}
u_{0}(x, t)=\sin x \\
u_{1}(x, t)=x t+\frac{1}{3} x t^{3}+\frac{1}{2} x t^{2} \cos x+t \cos x+t \sin x \cos x+\frac{1}{2} t^{2} \sin x, \\
u_{2}(x, t)=-t \cos x-t \sin x \cos x-\frac{1}{2} t^{2} \sin x-\cdots
\end{gathered}
$$

It is obvious that the last three terms in $u_{1}$ and the first three terms in $u_{2}$ are the self-canceling (noise terms). Keeping the remaining non-noise terms in $u_{1}$ leads to the exact solution of Equations (44)-(45), given by:

$$
u(x, t)=x t+\sin x \text {. }
$$

\section{2) Using ADSTM}

Proceeding as in Example 1, Equation (44) becomes:

$$
\begin{aligned}
\sum_{n=0}^{\infty} U_{n}(x, t)= & x t+\sin x+\frac{1}{3} x t^{3}+\frac{1}{2} x t^{2} \cos x+t \cos x+t \sin x \cos x \\
& +\frac{1}{2} t^{2} \sin x-S^{-1}\left[u S\left[\sum_{n=0}^{\infty} A_{n}+\left(\sum_{n=0}^{\infty} U_{n}(x, t)\right)_{x x x}\right]\right]
\end{aligned}
$$

The modified Sumudu decomposition method admits the of a modified recursive relation given by:

$$
\begin{gathered}
U_{0}(x, t)=x t+\sin x, \\
U_{1}(x, t)=\frac{1}{3} x t+\frac{1}{2} x t^{2} \cos x+t \cos x+t \sin x \cos x+\frac{1}{2} t^{2} \sin x \\
-S^{-1}\left[u S\left[A_{0}+\left(U_{0}(x, t)\right)_{x x x}\right]\right], \\
U_{k+1}(x, t)=-S^{-1}\left[u S\left[A_{k}+\left(U_{k}(x, t)\right)_{x x x}\right]\right], k \geq 1 .
\end{gathered}
$$

Consequently, we obtain: 


$$
\begin{gathered}
U_{0}(x, t)=x t+\sin x, \\
U_{1}(x, t)=0, \\
U_{k+1}(x, t)=0, k \geq 1 .
\end{gathered}
$$

The exact solution is given by:

$$
U(x, t)=x t+\sin x
$$

\section{Conclusions}

The main objective of this paper is to introduce a comparative study between modified Sumudu decomposition method and Homotopy perturbation method. The two methods are strong and effective methods that both hand approximations of higher reliability of series solution. A remarkable conclusion can made here. Modified Sumudu decomposition method gives rapid convergence of the series solution without showing noise terms. However, homotopy perturbation method provides the components of the series solution, where these components should have noise terms.

More importantly, the modified Sumudu decomposition method (MSDM) gives better efficiency in many cases, and this implies that the modified Sumudu decomposition method has an advantage over the homotopy perturbation method.

\section{References}

[1] Kumar, D., Singh, J. and Rathore, S. (2012) Sumudu Decomposition Method for Nonlinear Equations. International Mathematical Forum, 7, 515-521.

[2] Ahmed, S.A. and Elzaki, T. (2013) The Solution of Nonlinear Volterra Integro-Differential Equations of Second Kind by Combine Sumudu Transforms and Adomian Decomposition Method. International Journal of Advanced and Innovative Research, 2, 90-93.

[3] Ahmed, S.A. and Elzaki, T.M. (2014) A Comparative Study of Sumudu Decomposition Method and Sumudu Projected Differential Transform Method. World Applied Sciences Journal, 31, 1704-1709.

[4] Ahmed, S.E. and Elzaki, T.M. (2015) Solution of Heat and Wave-Like Equations by Adomian Decomposition Sumudu Transform Method. British Journal of Mathematics \& Computer Science, 8, 101-111. https://doi.org/10.9734/BJMCS/2015/9225

[5] Ahmed, S.A. (2014) Application of Sumudu Decomposition Method for Solving Burger's Equation. Advances in Theoretical and Applied Mathematics, 9, 2326.

[6] Eltayeb, H., Kilicman, A. and Mesloub, S. (2014) Application of Sumudu Decomposition Method to Solve Nonlinear Systems Volterra Integro-Differential Equations. Hindawi Publishing Corporation Abstract and Applied Analysis, 1-6.

[7] He, J.H. (1999) Homotopy Perturbation Technique. Computer Methods in Applied Mechanics and Engineering, 178, 257-262. https://doi.org/10.1016/S0045-7825(99)00018-3

[8] He, J.H. (1997) A New Approach to Nonlinear Partial Differential Equations. Communications in Nonlinear Science and Numerical Simulation, 2, 230-235. https://doi.org/10.1016/S1007-5704(97)90007-1 
[9] He, J.H. (1998) Approximate Analytical Solution for Seepage Flow with Fractional Derivatives in Porous Media. Computer Methods in Applied Mechanics and Engineering, 167, 57-68. https://doi.org/10.1016/S0045-7825(98)00108-X

[10] He, J.H. (1998) Approximate Solution of Nonlinear Differential Equations with Convolution Product Nonlinearities. Computer Methods in Applied Mechanics and Engineering, 167, 69-73. https://doi.org/10.1016/S0045-7825(98)00109-1

[11] Adomian, G. (1986) Nonlinear Stochastic Operator Equation. Academic Press, San Diego, CA.

[12] Adomian, G. (1984) Convergent Series Solution of Nonlinear Equations. Journal of Computational and Applied Mathematics, 11, 225-230.

https://doi.org/10.1016/0377-0427(84)90022-0

[13] Adomian, G. (1994) Solving Frontier Problems of Physics, The Decomposition Method. Kluwer, Boston. https://doi.org/10.1007/978-94-015-8289-6

[14] Adomian, G. (1994) Solution of Physical Problems by Decomposition. Computers and Mathematics with Application, 2, 145-154.

https://doi.org/10.1016/0898-1221(94)90132-5

[15] Adomian, G. and Rach, R. (1996) Modified Adomian Polynomials. Mathematical and Computer Modeling, 24, 39-46. https://doi.org/10.1016/S0895-7177(96)00171-9

[16] Wazwaz, A.M. (2006) The Modified Decomposition Method for Analytic Treatment of Differential Equations. Applied Mathematics and Computer, 173, 165-176. https://doi.org/10.1016/j.amc.2005.02.048

[17] Yildirim, A. (2009) On the Solution of the Nonlinear Korteweg-de Vries Equation by the Homotopy Perturbation Method. Communications in Numerical Methods in Engineering, 25, 1127-1136. https://doi.org/10.1002/cnm.1146

[18] Wazwaz, A.M. (1997) Necessary Conditions for the Appearance of Noise Terms in Decomposition Series. Applied Mathematics and Computers, 81, 265-274.

https://doi.org/10.1016/S0096-3003(95)00327-4 